Annali di Matematica pura ed applicata

(IV), Vol. CLXXVI (1999), pp. 49-55

\title{
Metrizable Extensions of Dynamical Systems from Function Algebras $\left.{ }^{*}\right)$.
}

\author{
JUAN J. FonT - MANUEL SANChIS
}

\begin{abstract}
Peters and Pennings introduced and studied in [9] and [11] a kind of extensions of dynamical systems induced by certain $C^{*}$-algebras, which they call tame extensions. In this paper we study the behaviour of metrizable tame extensions when some of the most important dynamical concepts related to the metric (such as sources, sinks, saddles, the shadowing property and distallity) are considered. We will confirm that these extensions can be troublesome in this context. We show, however, that sources and sinks are preserved under certain conditions.
\end{abstract}

\section{1. - Introduction.}

Throughout this paper $X$ will denote a compact metric Hausdorff space with metric $\varrho$. Let $\varphi: X \rightarrow X$ be a continuous mapping, and $\Sigma$ denote the natural numbers $N$ if $\varphi$ is not a homeomorphism, or the integers $Z$ otherwise. The triple $(X, \varphi, \Sigma)$ is a dynamical system.

Given $x \in X$, the set $\left\{\varphi^{k}(x): k \in \Sigma\right\}$ is called the $\varphi$-orbit of $x$ and is denoted by $\mathcal{O}_{\varphi}(x)$. $\varphi^{0}$ will stand for the identity on $X$.

A dynamical system $\left(X^{\prime}, \varphi^{\prime}, \Sigma\right)$ is said to be an extension of $(X, \varphi, \Sigma)$ if there is a continuous surjection $p: X^{\prime} \rightarrow X$ such that $p \circ \varphi^{\prime}=\varphi \circ p$. In this case, $(X, \varphi, \Sigma)$ is a factor of $\left(X^{\prime}, \varphi^{\prime}, \Sigma\right)$.

Let $C$ denote the complex numbers. Given a subset $A$ of $X$, we will denote by $|A|$ the cardinality of $A$. If $C(X)$ (resp. $B(X)$ ) are the algebras of continuous (resp. bounded) $C$-valued functions on $X$ endowed with the supremum norm $(\|\cdot\|)$ and $U$ is a $\Sigma$-invariant

$\left(^{*}\right)$ Entrata in Redazione il 24 febbraio 1997.

Indirizzo degli AA.: Departamento de Matemáticas, Universitat Jaume I, Campus Riu Sec E-12071, Castellón, Spain. E-mail: font@mat.uji.es; sanchis@mat.uji.es $58 \mathrm{~F} 13$.

1991 Mathematics Subject Classification: Primary 46L55; Secondary 34C35, 54H20,

Research partially supported by Generalitat Valenciana, (2223/94). 
$C^{*}$-algebra (that is, $C(X) \subset \mathcal{U} \subset B(X)$ and $f \circ \varphi^{k} \in \mathcal{U}$ provided $f \in \mathcal{U}, k \in \Sigma$ ), then there is an extension $(\widehat{X}, \widehat{\varphi}, \Sigma)$ of $(X, \varphi, \Sigma)$ where $\widehat{X}$ is the (compact) carrier space of $\mathcal{U}$ and $\widehat{\varphi}$ is the induced mapping given by $\widehat{\varphi}(\widehat{x})(f)=\widehat{x}(f \circ \varphi), f \in \mathcal{U}$. The mapping $p: \widehat{X} \rightarrow X$ is defined to be the restriction of pure states of $u$ to $C(X)$.

In [9] and [11], the authors introduced and studied this kind of extensions from function algebras. They say that $(\widehat{X}, \widehat{\varphi}, \Sigma)$ is a tame extension of $(X, \varphi, \Sigma)$ if there exists a $\Sigma$-invariant set $E \subset \mathcal{U}$, which generates $\mathcal{U}$ as a $C^{*}$-algebra, consisting of functions having at most finitely many points of discontinuity and such that $\mathcal{U}$ contains no characteristic functions of the form $\chi_{\{x\}}$. This latter property is equivalent ([9, Th.II9]) to the fact that $f(x) \in \Lambda(f ; x)$ for all $f \in \mathcal{U}, x \in X$, where $\Lambda(f ; x):=\{z \in C$ : there exists a net $\left(x_{i}\right) \subset X, x_{i} \rightarrow x, x_{i} \neq x$, such that $\left.f\left(x_{i}\right) \rightarrow z\right\}$.

The key of [9] and [11] is an alternative description of $\widehat{X}$. Namely, $\widehat{X}$ is the closure of the graph $\left\{\left(x,(f(x))_{f \in u}\right): x \in X\right\}$ with respect to the product topology. Furthermore, if $(\widehat{X}, \widehat{\varphi}, \Sigma)$ is a tame extension, then every point $\widehat{x} \in p^{-1}(x) \subset \widehat{X}$ has the form $\widehat{x}=$ $=\lim \left(x_{i},\left(f\left(x_{i}\right)\right)_{f \in u}\right)=\left(x,\left(\lambda_{f}\right)_{f \in u}\right)$, where $\left\{\left(x_{i}\right)\right\}_{i \in I}$ is a net in $X$ converging to $x$. The action of $\widehat{\varphi}$ on $\vec{X}$ is $\widehat{\varphi}(\widehat{x})=\left(\varphi(x),\left(\lambda_{f \circ \varphi}\right)_{f \in u}\right)$.

With no loss of generality, we will consider that $u$ is a $\Sigma$-invariant $C^{*}$-algebra generated by

$$
E=C(X) \bigcup\left\{f_{n} \circ \varphi^{k}: n \in N, k \in \Sigma\right\},
$$

where the functions $\left\{f_{n}\right\}_{n=1}^{\infty} \subset \mathcal{U}$ have unit norm. It is also possible (see [9, Remark II.3]) to realize $\widehat{X}$ as the closure of the graph

$$
\left\{\left(x,(f(x))_{f \in E}\right): x \in X\right\}
$$

Furthermore, $\widehat{X}$ is metrizable (see $[11, \mathrm{I} .5]$ ) and a metric $\widehat{\varrho}$ on $\widehat{X}$ is given by

$$
\widehat{\varrho}\left(\widehat{x}_{1}, \widehat{x}_{2}\right)=\varrho\left(x_{1}, x_{2}\right)+\sum_{n=1}^{\infty} \sum_{k \in \Sigma} \frac{\left|\widehat{f}_{n} \circ \widehat{\varphi}^{k}\left(\widehat{x}_{1}\right)-\widehat{f}_{n} \circ \widehat{\varphi}^{k}\left(\widehat{x}_{2}\right)\right|}{2^{|k|+n}},
$$

where $x_{i}=p\left(\widehat{x}_{i}\right),(i=1,2)$. For any $f \in \mathcal{U}, \widehat{f}$ will denote its Gel'fand transform.

Peters and Pennings proved that the following topological properties of dynamical systems are preserved under tame extensions: minimality, topological transitivity, strong (weak) topological mixing, having a dense set of (almost) periodic points and chaos. They showed that expansiveness and topological entropy are not always preserved. In [5], the authors focus on the study of recurrence problems in the context of tame extensions and show that a tame extension $(\widehat{X}, \widehat{\varphi}, \Sigma)$ preserves classical recurrence if $(X, \varphi, \Sigma)$ satisfies certain new (stronger) kind of recurrence.

In this paper we study the behaviour of metrizable tame extensions when some of the most important dynamical concepts related to the metric (such as sources, sinks, saddles, the shadowing property and distallity) are considered. We will confirm that these extensions can be troublesome in this context (see [11] or [10, Section III]). We show, however, that sources and sinks are preserved under certain conditions. 


\section{2. - Sources, sinks and saddles.}

Definition 1. - Let $X$ be a compact metric space and let $\varphi: X \rightarrow X$ be a homeomorphism. The local stable set of $\varphi$ at $x \in X$ for $\delta \geqslant 0$ is defined as follows:

$$
W^{s}(x, \delta)=\left\{y \in X: \varrho\left(\varphi^{n}(x), \varphi^{n}(y)\right) \leqslant \delta \text { for } n \geqslant 0\right\} .
$$

The local unstable set of $\varphi$ at $x \in X$ for $\delta \geqslant 0$ is defined to be:

$$
W^{u}(x, \delta)=\left\{y \in X: \varrho\left(\varphi^{-n}(x), \varphi^{-n}(y)\right) \leqslant \delta \text { for } n \geqslant 0\right\} .
$$

DEFINITION 2. - A point $x \in X$ is called $a$ source for the homeomorphism $\varphi$ if $W^{s}(x, \alpha)=\{x\}$ for some positive real number $\alpha$. It is called a $\operatorname{sink}$ for $\varphi$ if it is a source for $\varphi^{-1}$. If it is neither a source nor a sink for $\varphi$, then it is called a saddle point for $\varphi$.

THEOREM 1. - Let $X$ be a compact metric space, $(X, \varphi, \boldsymbol{Z})$ a dynamical system and $(\widehat{X}, \widehat{\varphi}, \boldsymbol{Z})$ a tame extension such that the $C^{*}$-algebra $u$ is generated by $E=C(X) \cup$ $\cup\left\{f_{1}, \ldots, f_{n}\right\}$. Let $x \in X$ be a source (resp. a sink) for $\varphi$ such that $\left|\Lambda\left(f_{i} ; x\right)\right|<\infty$ for $i=$ $=1, \ldots, n$. Then every $\widehat{x}$ in $p^{-1}(x)$ is a source (resp. a sink) for $\widehat{\varphi}$.

Proof. - If $x \in X$ is a source for $\varphi$, then there exists $\alpha^{\prime}>0$ such that $W^{s}\left(x, \alpha^{\prime}\right)=$ $=\{x\}$. Hence, if $y \in X$ is different from $x$, then there exists $m \in N$ such that $\varrho\left(\varphi^{m}(x), \varphi^{m}(y)\right)>\alpha^{\prime}$. Consequently, $\widehat{\varrho}\left(\widehat{\varphi}^{m}(\widehat{x}), \widehat{\varphi}^{m}(\widehat{y})\right)>\alpha^{\prime}$ for all $\widehat{x} \in p^{-1}(x)$ and all $\widehat{y} \in p^{-1}(y)$. That is, $\widehat{y} \notin W^{s}\left(\widehat{x}, \alpha^{\prime}\right)$ for all $\widehat{x} \in p^{-1}(x)$ and all $\widehat{y} \in p^{-1}(y)$.

Let us now fix $\widehat{x}^{\prime} \in p^{-1}(x)$. Consider $\widehat{x} \in p^{-1}(x)$ with $\widehat{x}^{\prime} \neq \widehat{x}$. Since, by hypothesis, $\Lambda\left(f_{i} ; x\right)$ has finite cardinality for $i=1, \ldots, n$, we can consider

$$
s=\min \left\{\left|\widehat{f}_{i}\left(\widehat{x}^{\prime}\right)-\widehat{f}_{i}(\widehat{x})\right| \neq 0: \widehat{x} \in p^{-1}(x), i=1, \ldots, n\right\} .
$$

Hence

$$
\widehat{\varrho}\left(\widehat{x}, \widehat{x}^{\prime}\right) \geqslant \frac{\left|\widehat{f}_{i^{\prime}}(\widehat{x})-\widehat{f}_{i^{\prime}}\left(\widehat{x}^{\prime}\right)\right|}{2^{i^{\prime}}}
$$

for some $i^{\prime} \in\{1, \ldots, n\}$. Thus $\hat{\varrho}\left(\widehat{x}, \widehat{x}^{\prime}\right) \geqslant s / 2^{n}$. As a consequence, if we take $\alpha=$ $=\min \left\{\alpha^{\prime}, s / 2^{n}\right\}$, then $W^{s}\left(\widehat{x}^{\prime}, \alpha\right)=\left\{\hat{x}^{\prime}\right\}$.

It is easy to verify that if $\left|\Lambda\left(f_{i} ; x\right)\right|=\infty$ for some $i \in\{1, \ldots, n\}$, then Theorem 1 may fail.

The following example shows that Theorem 1 is not valid for saddle points.

EXAMPLE 1. - Let $X$ be the interval $[-1,1]$ and let $\varphi: X \rightarrow X$ be a homeomorphism defined as

$$
\varphi(x)= \begin{cases}x, & x \in[-1,0], \\ \sqrt{x}, & x \in[0,1] .\end{cases}
$$


It is easy to check that 0 is neither a source nor a sink for $\varphi$ since, given $\alpha>0$ and $y \in\left[-1,0\left[\right.\right.$ with $\varrho(0, y)<\alpha$, then $y \in W^{s}(0, \alpha) \cap W^{u}(0, \alpha)$. Hence, 0 is a saddle point for $\varphi$. Now we define the following function on $X$ :

$$
f(x)= \begin{cases}0, & x \in[-1,0[ \\ 1, & x \in[0,1]\end{cases}
$$

Consider now the tame extension $(\widehat{X}, \widehat{\varphi}, Z)$ induced by the $C^{*}$-algebra generated by $E=C(X) \cup\left\{f \circ \varphi^{k}: k \in Z\right\}$ ([9, Corollary II.12]). Let $\widehat{0}=\left(0,\left(\lambda_{g}\right)_{g \in E}\right)$ such that $\lambda_{g}=1$ for $g=f \circ \varphi^{k}, k \in \boldsymbol{Z}$. We claim that $\hat{0}$ is a source for $\widehat{\varphi}$, which implies that it is not a saddle point for $\widehat{\varphi}$. To this end, take $\widehat{y}_{1} \in p^{-1}\left(y_{1}\right), y_{1} \in\left[-1,0\left[\right.\right.$. Thus, $\widehat{y}_{1}=\left(y_{1}, \ldots, 0,0,0, \ldots\right)$ and, consequently,

$$
\widehat{\varrho}\left(\widehat{0}, \widehat{y}_{1}\right) \geqslant \frac{\left|\widehat{f}(\widehat{0})-\widehat{f}\left(\widehat{y_{1}}\right)\right|}{2^{1}}=\frac{1}{2} .
$$

In like manner, we infer that $\widehat{\varrho}\left(\hat{0}, \hat{0}^{\prime}\right) \geqslant 1 / 2$ where $\hat{0}^{\prime}=(0, \ldots, 0,0,0, \ldots)$.

On the other hand, choose $\left.\left.\widehat{y}_{2} \in p^{-1}\left(y_{2}\right), y_{2} \in\right] 0,1\right]$. Hence, if we fix $\alpha=1 / 3$, then there exists $n\left(y_{2}\right) \in N$ such that $\varrho\left(\varphi^{n\left(y_{2}\right)}(0), \varphi^{n\left(y_{2}\right)}\left(y_{2}\right)\right)>1 / 3$. Consequently, $\widehat{\varrho}\left(\widehat{0}, \widehat{y}_{2}\right)>$ $>1 / 3$. Summing up, we deduce that $W^{s}(\hat{0}, 1 / 3)=\{\hat{0}\}$, that is, $\widehat{0}$ is a source for $\widehat{\varphi}$.

\section{3. - The shadowing property.}

Definition 3. - Let $(X, \varrho)$ be a compact metric space and let $\varphi: X \rightarrow X$ be a homeomorphism. A sequence of points $\left\{x_{i}: i \in(a, b)\right\}(-\infty \leqslant a<b \leqslant \infty)$ is called a $\delta$-pseudo-orbit for $\varphi$ if $\varrho\left(\varphi\left(x_{i}\right), x_{i+1}\right)<\delta$ for $i \in(a, b-1)$. A sequence $\left\{x_{i}\right\}$ is called to be $\varepsilon$-traced by $x \in X$ if $\varrho\left(\varphi^{i}(x), x_{i}\right)<\varepsilon$ for $i \in(a, b)$. It is said that $\varphi$ has the pseudoorbit tracing property (POTP) or the shadowing property provided that, for each $\varepsilon>0$, there exists $\delta>0$ such that every $\delta$-pseudo-orbit for $\varphi$ is $\varepsilon$-traced by some point of $X$.

The investigation of the pseudo-orbits is very important in connection with the calculation of the orbits by computers, since they can calculate only pseudo-orbits. In the following we study the behaviour of the POTP for the identity map under tame extensions. Let us first recall an interesting result which reduces this problem to a purely topological one:

THEOREM 2 ([1, Remark 4.30]). - Let $X$ be a compact metric space. Then the identity map $\varphi^{0}: X \rightarrow X$ has the POTP if and only if $X$ is totally disconnected.

Hence, since we do not consider spaces with isolated points (otherwise $u$ contains characteristic functions (see the definition of a tame extension)) and the only totally disconnected compact metrizable space without isolated points is, up to a topological homeomorphism, the Cantor set $\mathcal{C}$ (See $[4,6.2 . \mathrm{A}(\mathrm{c})]$ ), we will focus on the dynamical system $\left(\mathcal{C}, \varphi^{0}, Z\right)$.

First, let us recall several facts about the Cantor set. The two point discrete space will be denoted by $D$ and will be identified with the subspace $\{0,1\}$ of the real line. The 
cartesian product $\mathcal{C}=\prod_{n \in N} D_{n}$ where $D_{n}=D$ for every $n \in N$ is called the Cantor set. The Cantor set is homeomorphic to a subspace of the real line: the set of all real numbers of the segment $[0,1]$ that have a tryadic expansion in which the digit 1 does not occur.

On the other hand, a well-known theorem $([8,15.8$, Th. 4$])$ states that every compact metric space is the continuous image of the Cantor set. Thus, the dynamical system $\left(\mathcal{C}, \varphi^{0}, \boldsymbol{Z}\right)$ is an extension of $\left([0,1], \varphi^{0}, \boldsymbol{Z}\right)$. This shows that the POTP is not, in general, inherited by factors. In the context of tame extensions the situation is not better as the following example shows.

EXAMPLE 2. - Let $X$ be the unit interval $[0,1]$ and let $S:=0,1[\cap \boldsymbol{Q}$, where $\boldsymbol{Q}$ stands for the rational numbers. For each $s \in S$, we define a function $f_{s}$ as:

$$
f_{s}(x)= \begin{cases}0, & x<s \\ 1, & x \geqslant s\end{cases}
$$

Consider the tame extension $\left(\widehat{X}, \widehat{\varphi^{0}}, Z\right)$ of $\left(X, \varphi^{0}, Z\right)$ induced by the $C^{*}$-algebra generated by $E=C(X) \cup\left\{f_{s}: s \in S\right\}$ (see [9, Corollary II.12]). We shall prove that $\widehat{X}$ is a totally disconnected metric space. Hence, by Theorem $2,\left(\widehat{X}, \widehat{\varphi^{0}}, Z\right)$ satisfies the POTP (it is a routine matter to verify that $\widehat{\varphi^{0}}: \widehat{X} \rightarrow \widehat{X}$ is the identity map), but its factor $\left(X, \varphi^{0}, Z\right)$ does not.

In order to check that $\widehat{X}$ is totally disconnected, let us recall (see section 1) that $\widehat{X}$ can be realized as the closure of the graph $\left\{\left(x,(f(x))_{f \in E}\right): x \in X\right\}$ in $X \times \prod_{f \in E} \overline{f(X)}$.

Fix $\left(x_{0},\left(\lambda_{f}\right)_{f \in E}\right) \in \widehat{X}\left(x_{0} \neq 0\right.$ and $\left.x_{0} \neq 1\right)$. For each $n \in N$, we choose two points $s_{n}, r_{n}$ in $] x_{0}-1 / n, x_{0}+(1 / n)\left[\cap S\right.$ with $s_{n}<x_{0}<r_{n}$. Let us distinguish two cases:

- If $x_{0} \notin S$, we define

$$
\left.U_{n}:=\right] x_{0}-\frac{1}{n}, x_{0}+\frac{1}{n}\left[\times\left\{f_{s_{n}}\left(x_{0}\right)\right\} \times\left\{f_{r_{n}}\left(x_{0}\right)\right\} \times \prod_{f \in E \backslash\left\{f_{s_{n}}, f_{r_{n}}\right\}} \overline{f(X)} .\right.
$$

Let $W_{n}:=U_{n} \cap \widehat{X}$. A moment's reflection shows that $W_{n}=\left[s_{n}, r_{n}\right] \times\left\{f_{s_{n}}\left(x_{0}\right)\right\} \times$ $\times\left\{f_{r_{n}}\left(x_{0}\right)\right\} \times \prod_{f \in E \backslash\left\{f_{n_{n}}, f_{r_{n}}\right\}} \overline{f(X)}$, which implies that $W_{n}$ is a clopen subset of $\widehat{X}$.

- If $x_{0} \in S$, we define

$$
\left.U_{n}:=\right] x_{0}-\frac{1}{n}, x_{0}+\frac{1}{n}\left[\times\left\{\lambda_{f_{x_{0}}}\right\} \times\left\{f_{8_{n}}\left(x_{0}\right)\right\} \times\left\{f_{r_{n}}\left(x_{0}\right)\right\} \times \prod_{f \in E \backslash\left\{f_{s_{n}}, f_{r_{n}}, f_{x_{0}}\right\}} \overline{f(X)} .\right.
$$

Let $W_{n}:=U_{n} \cap \widehat{X}$. Reasoning as above, we deduce that $W_{n}$ is a clopen subset of $\widehat{X}$ too.

Since it is clear that

$$
\bigcap_{n \in N} W_{n}=\left(x_{0},\left(\lambda_{f}\right)_{f \in E}\right),
$$

we infer that $\left(x_{0},\left(\lambda_{f}\right)_{f \in E}\right)$ is the intersection of all clopen subsets of $\widehat{X}$ which contain it. 
As the (connected) component of a point in a compact space is just the intersection of all clopen subsets containing it (see [4, Theorem 6.1.23]), the component of $\left(x_{0},\left(\lambda_{f}\right)_{f \in E}\right)$ is the singleton $\left\{\left(x_{0},\left(\lambda_{f}\right)_{f \in E}\right)\right\}$.

Finally, to obtain the totally disconnectness of $\widehat{X}$, we only have to check that the components of the remaining two points of $\widehat{X}$, namely $\left(0,(f(0))_{f \in E}\right)$ and $\left(1,(f(\mathbf{1}))_{f \in E}\right)$, are singletons. Indeed, this follows from the fact that every connected Tychonoff space containing at least two point cannot be countable ([4, Corollary 6.1.3]).

The following theorem characterizes when a tame extension of $\left(\mathcal{C}, \varphi^{0}, \boldsymbol{Z}\right)$ has the POTP property.

Theorem 3. - Let $\left(\widehat{\mathcal{C}}, \widehat{\varphi^{0}}, \boldsymbol{Z}\right)$ be a tame extension of $\left(\mathcal{C}, \varphi^{0}, \boldsymbol{Z}\right)$ induced by a $C^{*}$-algebra $U$. Then $\left(\widehat{\mathcal{C}}, \widehat{\varphi^{0}}, \boldsymbol{Z}\right)$ has the POTP if and only if $\mathcal{U}=C(\mathcal{C})$.

Proof. - Suppose that $\left(\widehat{\mathcal{C}}, \widehat{\varphi^{0}}, \boldsymbol{Z}\right)$ has the POTP. By the Gel'fand-Naimark Theorem, the $C^{*}$-algebras $u$ and $C(\widehat{\mathcal{C}})$ are isometrically isomorphic. Hence, since $u$ has no characteristic functions (see the definition of a tame extension), we infer that $\overline{\mathcal{C}}$ has no isolated points. Now, by Theorem 2 , the compact space $\widehat{\mathcal{C}}$ is homeomorphic to the Cantor set $\mathcal{C}$. Finally the Gel'fand-Kolmogoroff Theorem entails that $C(\widehat{\mathcal{C}})$ and $C(\mathcal{C})$ are isometrically isomorphic (see [7]).

The converse is clear since the equality $u=C(\mathcal{C})$ yields $\left(\widehat{\mathcal{e}}, \widehat{\varphi^{0}}, \boldsymbol{Z}\right)=$ $=\left(\mathfrak{C}, \varphi^{0}, \boldsymbol{Z}\right)$.

Theorem 3, consequently, provides numerous examples of tame extensions which do not preserve the POTP.

\section{4. - Distallity.}

Definition 4. - Let $\varphi: X \rightarrow X$ be a homeomorphism of a compact metric space. It is said that $\varphi$ is distal if inf $\left\{\varrho\left(\varphi^{k}(x), \varphi^{k}(y)\right): k \in \boldsymbol{Z}\right\}=0$, then $x=y$.

Two points $x, y \in X$ are said to be proximal if for every $\varepsilon>0$, there exists $k \in \boldsymbol{Z}$ such that $\varrho\left(\varphi^{k}(x), \varphi^{k}(y)\right)<\varepsilon$.

It is clear that a dynamical systen is distal if and only if it contains no pair of proximal points. The following theorem shows that, in many cases, tame extensions cannot be distal.

THEOREM 4. - Let $X$ be a compact metric space, $(X, \varphi, \boldsymbol{Z})$ a dynamical system and $(\widehat{X}, \widehat{\varphi}, \boldsymbol{Z})$ a tame extension such that the $C^{*}$-algebra $\mathcal{U}$ is generated by $E=C(X) \cup$ $\cup\left\{f_{1}, \ldots, f_{n}\right\}$. If $(X, \varphi, \boldsymbol{Z})$ has no periodic points, then $(\widehat{X}, \widehat{\varphi}, \boldsymbol{Z})$ has, at least, a pair of proximal points.

Proof. - Since $\left\{f_{1}, \ldots, f_{n}\right\}$ is finite and each function $f_{i}(i=1, \ldots, n)$ has at most finitely many points of discontinuity, we shall prove the case $n=1$ (i.e., when $E=$ $=C(X) \cup\{f\}$ ), assuming further that $f$ has an only point of discontinuity $x_{0}$. The general case follows from the same arguments.

The discontinuity of $f$ at $x_{0}$ implies that $p^{-1}\left(x_{0}\right)$ contains at least two (distinct) 
points $\widehat{x}$ and $\widehat{y}$ (induced by two sequences converging to $x_{0}$ respectively). Since the function $f$ is assumed to have unit norm (see sect. 1) and $x_{0}$ is not a periodic point for $(X, \varphi, \boldsymbol{Z})$, that is, $\varphi^{k}\left(x_{0}\right) \neq \varphi^{k^{\prime}}\left(x_{0}\right)$ whenever $k \neq k^{\prime}$, a moment's reflection shows that

$$
\hat{\varrho}\left(\widehat{\varphi}^{k}(\widehat{x}), \widehat{\varphi}^{k}(\widehat{y})\right)=\frac{\left|\hat{f} \circ \widehat{\varphi}^{k-k}\left(\widehat{x}_{1}\right)-\widehat{f} \circ \widehat{\varphi}^{k-k}\left(\widehat{x}_{2}\right)\right|}{2^{1+|-k|}} \leqslant \frac{2}{2^{1+1-k \mid}}
$$

for every $k \in \boldsymbol{Z}$. As a consequence we infer that $\hat{x}$ and $\hat{y}$ are proximal.

REMARK 1. - In spite of the above theorem, not all tame extensions fail to be distal. For example, any tame extension of $\left(X, \varphi^{0}, Z\right)$ is distal since $\widehat{\varphi^{0}}: \widehat{X} \rightarrow \widehat{X}$ is the identity map.

\section{REFERENCES}

[1] N. AokI, Topological Dynamics (K. Morita, J. Nagata, Eds.), Topics in General Topology. Elsevier Science Publishers (1989), pp. 625-740.

[2] L. S. Bl.OCK - W. A. CopPEL, Dynamics in One Dimension, Lecture Notes in Math., 1513 (1992).

[3] L. E. J. Brocwer, On the structure of perfect sets of points, Proc. Akad. Amsterdam, 12 (1910), pp. 785-794.

[4] R. Engelking, General Topology, Polish Scientific Publishers, Warszawa (1977).

[5] J. J. FONT - M. SANCHIS, On extensions of dynamical systems by function algebras. Submitted.

[6] H. Furstenberg, Recurrence in Ergodic Theory and Combinational Number Theory, Princeton Univ. Press, Princeton, N.J. (1981).

[7] L. Gillman - M. Jerison, Rings of Continuous Functions, Van Nostrand, New York (1960).

[8] K. KuRatowski, Wstep do Teorii Mnogosci i Topologii, Panstowore Wydawnicton Naukowe, Warszawa (1961).

[9] T. Pennings - J. Peters, Dynamical systems from function algebras, Proc. A.M.S., 105 (1989), pp. 80-86.

[10] T. Pennings, Extensions of Dynamical Systems, Doctoral Dissertation, Iowa State University (1987).

[11] J. Peters - T. Pennings, Chaotic extensions of dynamical systems by function algebras, J. Math. Anal. Appl., 159 (1991), pp. 345-360. 